\title{
Thermogenesis, aging and obesity in the LA/Ntul//-cp (corpulent) rat
}

\begin{abstract}
Obesity and mild to moderate impaired carbohydrate tolerance develops in the obese phenotype of the LA/Ntul//-cp (corpulent) rat strain by 6 weeks of age. Groups $(n=12-$ 20 rats/phenotype] of female congenic lean and obese LA/Ntul//-cp (corpulent) rats were fed ad libitum standardized Purina \#5012 diet ad house water for 4, 14, or 24 months of age. Measures of Body weight (BW) and of resting oxygen consumption (RMR; VO2) at thermal neutrality and for up to 45 minutes of $\left(4^{\circ} \mathrm{C}\right)$ spontaneous cold exposure, and norepinephrine-stimulated thermogenesis (100 ug. s.c.) were determined at each age. Body weights of lean rats increased only gradually during the study $(\mathrm{p}=\mathrm{n} . \mathrm{s}$.$) , but weights of$ obese phenotype were $\sim 2$-fold greater at 4 months $(\mathrm{p}=<0.05), 14$ months $(\mathrm{p}-<0.01)$, and $\sim 3.5$ to 4 -fold greater at 24 months of age $(p=<0.01)$. Resting metabolic rates in the lean phenotype trended to decrease modestly by 24 months $(\mathrm{p}=<0.05)$ and were greater than resting metabolic rates in the obese phenotype at all ages studied $(\mathrm{p}=<0.05)$. Cold exposure at $4{ }^{\circ} \mathrm{C}$ resulted in a dramatic $\sim 5$-fold increase in the oxygen consumption after 5 minutes in 4-month-old rats, which subsided to $\sim 2 \mathrm{x}$ resting metabolic rate within 15 minutes and remained constant thereafter in the lean phenotype. In 14-months old lean rats the increase in oxygen consumption at 5 minutes averaged $\sim 4$-fold increase over resting levels and returned to $\sim 2 \mathrm{x}$ resting levels from 15 to 45 minutes $4^{\circ} \mathrm{C}$ exposure. In 24 -month-old lean rats however, the cold induced increases in metabolism were markedly diminished to only $\sim 3 \mathrm{x}$ resting metabolic rates at 5 minutes cold exposure but remained similar to the other lean age groups thereafter. In the obese phenotype, the peak responses at 5 minutes were lower than those of lean rats at 5 minutes in 4- and 14-month-old rats and were significantly impaired in 24-month-old rats at all times measured. Norepinephrine resulted in a $>1.5$-fold increase in oxygen consumption in the lean phenotype at all ages studied, while in obese rats norepinephrine resulted in a 1.4-fold increase in oxygen consumption at 4 months of age with non-significant $\sim 1.1$ to 1.2 -fold increases at the two older ages. In both phenotypes, the resting and the norepinephrine-stimulated oxygen consumption responses tended to decrease with increasing age. The results of this study demonstrate that the body weights of the obese phenotype are significantly greater than their lean littermates throughout the age spectrum studied, and that both resting metabolic rates, $4^{\circ} \mathrm{C}$ cold, and norepinephrinestimulated thermogenesis are decreased in the obese compared to the lean phenotype and become further decreased with advancing age.
\end{abstract}

Keywords: obesity, thermogenesis, congenic rat
Volume II Issue 2 - 202 I

\author{
Orien L Tulp, ${ }^{1-3}$ George P Einstein ${ }^{1-3}$ \\ 'University of Science Arts and Technology, Montserrat \\ ${ }^{2}$ Colleges of Medicine and Graduate Studies, The University of \\ Health and Humanities, Virgin Islands, \\ ${ }^{3}$ The Einstein Medical Institute, North Palm Beach FL, USA
}

Correspondence: Dr Orien L Tulp, University of Science Arts and Technology, Aspen Business Park, 5400 Ward Road, Building III, Suite 150, Arvada, CO, 80002. USA, Tel 0I-303-352-0I I5, Fax 0I-303-37I-0I I7, Email o.tulp@usat.edu

Received: February 25, 2021 | Published: March 08, 2021

\section{Introduction}

The burgeoning incidence of obesity and overweight conditions with their common metabolic sequalae present a significant concern and potential epidemic for much of Western society and occurs due at least in part to an imbalance between caloric intake and energy expenditure occurring over an extended duration., ${ }^{1,2}$ Once thought to be a phenomenon more common when approaching middle age, the onset of excess adiposity is now more prevalent in children and adolescents than in past generations, where it poses a potentially greater threat to development of the pathophysiologic sequalae commonly associated with excess weight gain earlier in the lifespan than in past generations. Thus, the potential negative impact on human health and longevity may now become exaggerated and more difficult to reverse when therapeutic regimens are applied. Additionally, the epidemic is gaining traction throughout the age spectrum as changing lifestyles trend toward more sedentary employment options combined with a broader assortment of dietary preferences available to an everchanging society. ${ }^{1-3}$

This phenomenon of increasing incidence of adiposity appears to be multifactorial in nature, including factors of dietary selection, changing exercise patterns, metabolic factors, and genetic predisposition. $^{3-5}$ As the incidence of obesity and overweight conditions continue to increase in Western society, so does the incidence of NIDDM, hypertension, and other significant comorbid age- and obesity-related conditions. The independent variables of age and genetic predisposition are more difficult to assess in human than in congenic animal populations however due at least in part to the wide genetic variability and variations in lifestyle present in the general population, but may be minimized in a congenic rodent model that can express an obesity trait independent of dietary factors. ${ }^{6-8}$

The LA/Ntul//-cp (corpulent) rat is a congenic animal model, developed from the LA/N strain at the NIH by Hansen and further characterized by Michaelis \& Tulp. ${ }^{8}$ Obesity occurs in this strain as the result of an autosomal recessive trait, originally derived from the Koletsky rat by Hansen and thus appears in $\sim 25 \%$ of the offspring of heterozygous breeding pairs for the (-cp) trait. ${ }^{6,9}$ Obesity and mild to moderate impaired carbohydrate tolerance develop in the obese phenotype of the LA/Ntul/l-cp (corpulent) rat strain by 6 weeks of age. ${ }^{8,10}$ The obese phenotype exhibits a number of endocrine-related characteristics by early adulthood, including hyperinsulinemia, 
hyperamylinemia, hyperlipidemia, and mild to moderate glucose intolerance, but obese animals remain normotensive and nondiabetic. ${ }^{10-15}$ Obese offspring have rarely been observed to reproduce due to endocrinopathies that as yet remain unclear. ${ }^{8}$

The animals of the current study were derived following the 12th backcross at the NIH by Hansen thereby establishing the congenic criterion for the colony and virtually eliminating experimental variability within the strain with the exception of the obese $(-c p)$ characteristic expressed in the obese phenotype ${ }^{6-9}$ Because the obesity phenotype is expressed as an autosomal recessive trait it occurs with great consistency in $-25 \%$ of the male and female offspring of heterozygous breeders, and thus is an excellent model to investigate the independent variables of age and phenotype on metabolic factors likely to be contributory or causative to the predisposition of obesity and overweight conditions independent of NIDDM. ${ }^{6,9}$ The LA/N background strain was noted for its longevity by Hansen and in studies by Tulp indicate that this strain ranks among the most long-lived of the obese rodent models yet reported, with some animals surviving beyond 4 years under standard laboratory conditions. Lean animals have a normal thyroidal response to diet and cold environment, while the dietary and environmental responses in obese animals exhibiting the $(-c p)$ trait have been shown to be impaired. ${ }^{12-15}$ Except for the obese trait, biologic variability between offspring of different litters is virtually absent due to the congenic and specific pathogen-free (SPF) status of this strain and is limited to the potential heterozygosity within the lean littermates of the strain, some of which may carry a homozygous lean trait (--/--) and some a heterozygous $(-/-c p)$ lean trait. In contrast, all obese littermates are homozygous $(-c p /-c p)$ for the obese characteristic. All breeding pairs consisted of litter-proven heterozygous carriers for the obese trait as described above. ${ }^{14}$ The purpose of the present study was to determine the effect of aging and the genetic predisposition for obesity on resting, environmental- and noradrenaline stimulated thermogenesis across much of the age span to 24 months of age.

The physiologic elements of thermogenesis in mammalian species involve multiple organs and tissues and include both metabolic and neuroendocrine contributions to bring about the thermic responses to diet and environment. ${ }^{3,4,15-17}$ In other studies, both thyroidal and sympathetic nervous system (SNS) have been shown to participate in thyroidal-mediated actions for longer term permissive responses, and the SNS for acute, short term responses with a likely faciliatory interaction of unclear proportions between the two systems. In the thyroidal element, T4 must first be deiodinated to T3, to be active via an intracellular thyroxine-3,5,3' deiodinase (outer ring deiodinase) in response to diet and environment to form the physiologically active form of thyroxine, T3. In contrast T4 can also undergo inner ring deiodination via thyroxine-3',5,3-deiodination to form 'reverse' T3, triiodothryine-3', 5',3-T3, a metabolically inactive form of thyroid hormone in response to starvation and food depravation. ${ }^{3,16-20}$ Dietary $\mathrm{CHO}$ availability is among the major nutritional factors that triggers the switch from the 'fed pathway forming T3 vs. the 'fasting' pathway resulting in formation of metabolically inactive "reverse T3' (rT3), while T4 generation and circulating concentrations typically remain unchanged. ${ }^{3,8}$ While nuclear binding sites can bind T4, T3 and rT3, the intracellular nuclear binding affinity favors $\mathrm{T} 3$ over other thyroidal metabolites. ${ }^{18}$ In addition, the plasma half-life (T1/2) of T4 to T3 was found to be significantly longer in obese than in lean animals, indicative of a decreased intracellular activation of thyroid activity, and decreases in activity of intracellular thyroid dependent metabolic processes including mitochondrial a-glycerophosphate dehydrogenase activity ${ }^{21-27}$ The nutritional modulation of iodothyronine-5' deiodinase activity in peripheral tissues of lactating dams where differential responses were reflective of organ- and tissue-specific 5'deiodinase activity in BAT and other tissues and contributes to T3 content of milk for the developing offspring while conserving T3 generation in selected maternal tissues. ${ }^{27}$

Other endocrine linked alterations prevalent in the obese phenotype include elevations in both plasma insulin and amylin concentrations, and the progressive development of decreased receptor mediated sensitivity to these hormones. ${ }^{24,26}$ Amylin is also critical in mediating gastric emptying, contributing to appetite and feeding behavior in man and animals. ${ }^{11}$ Insulin contributes to macronutrient metabolism including aspects of carbohydrate, protein and lipid metabolism. ${ }^{16}$ Glut-4, important in facilitating glucose uptake in white adipose tissue and skeletal muscle, primary biological sites for insulin resistance. ${ }^{20-24}$ In addition, hyperinsulinemia is also a hormonal contributor to both protein synthesis and protein degradation, both of which have been observed to be associated with a decreased rate of protein turnover in the obese animals of this strain, resulting in an economy of protein requirements. ${ }^{24}$ When lean animals were fed a protein restricted diet, their linear and lean tissue growth was proportionately decreased, while the obese animals were fed the same protein restricted diets linear and muscle growth were unaffected, and the animals growth and development were equivalent to normally fed obese animals, ${ }^{25}$ consistent with insulinogenic normalization of nitrogen balance in obese Zucker rats fed the same protein restricted diets demonstrated a similar pattern of growth and development, and similarity of endocrinologic responses to the obese gene. ${ }^{26,28}$ When 6-week-old genetically preobese Zucker rats received a hypothalamic implant obtained from homozygous lean 14-day gestational age fetuses, the endocrinologic and thermic responses became normalized and remained similar to those of lean littermate animals into adulthood. ${ }^{29}$

Insulin has also been shown to play a permissive role in adaptive thermogenesis in various tissues including BAT, where it facilitates glucose uptake via insulin dependent GLUT transporters resulting in a biochemical cascade of oxidative metabolism and the generation of reducing equivalents needed for mitochondrial oxidation. Insulin resistance, as commonly occurs in an obese state would be expected to impair glucose uptake in muscle and adipose tissue including BAT among other tissues and would likely impair the glucosemediated thermic bioenergetic responses to diet, environment and neuroendocrine activation, resulting in an improved efficiency of caloric efficiency, and thereby contribute to the obese state. ${ }^{14,15}$ The obese phenotype of the LA/Ntul//-cp rat has been shown to exhibit mild to moderate glucose intolerance, typical of progressive insulin resistance with ageing but remain non-diabetic $c^{10,11,13,14}$ whereas other rodent models expressing the same $-c p$ or other fatty traits including the $-f a$ and $-o b$ trait also develop non-insulin dependent diabetes mellitus. (Type-2 diabetes or NIDDM) early in life. ${ }^{7,30-37}$ In the present study, we sought to determine the effects of aging on environmental and neuroendocrine-stimulated norepinephrine-stimulated thermogenesis via cold exposure and administration of a standardized dose of exogenous norepinephrine (NE) on thermogenesis in the lean LA/Ntul//-cp rat. This is a highly useful animal model, in that the only genetic difference between the lean and obese phenotypes is the presence or absence of the expression of the $-c p$ trait, and the metabolic changes that may be secondarily linked to the obesity.

\section{Materials and methods}

Groups ( $n=12-20$ rats/group) of congenic specific pathogen free (SPF) lean and obese littermate female offspring of heterozygous breeders in the Drexel colony were obtained postweaning at 6 weeks of age. All animals were placed in plastic cages in littermate pairs, 
with cages lined with pine shavings on a reverse light cycle (dark 0800-2000 hrs.) in a temperature-controlled environment $\left[22+/-1^{\circ} \mathrm{C}\right]$ and $40-60 \%$ relative humidity from 6 weeks of age. All animals consumed the same Purina \#5012 diet and house water ad libitum, throughout the study. This diet provided 55.6\% CHO. $22.5 \%$ protein, $4.5 \%$ fat, $4.6 \%$ crude fiber, $6 \%$ ash, and $12 \%$ essential vitamins and minerals, and had an energy density of $3.4 \mathrm{kcal} / \mathrm{gram}$ (14.2 kjoules/ gram). All housing and laboratory procedures were in accordance with current NIH, AVMA and Institutional Animal care and Use Committee guidelines. ${ }^{38}$

Measures of animal body weight were obtained with an Ohaus animal balance to the nearest gram at weekly intervals as described by Vedula et al. ${ }^{14,36}$ Measures of resting thermogenesis were obtained at thermal neutrality $\left(29^{\circ} \mathrm{C}\right)$ as described previously. Briefly, animals were individually placed in a sealed closed circuit plexiglass chamber canister of $1 \mathrm{c}$.f. volume, connected to a spirometer apparatus, where the chamber temperature is maintained in a water bath at $29+/-0.5^{\circ} \mathrm{C}$ throughout the measurements, except as noted. The respiratory $\mathrm{CO}_{2}$ generated was removed in toto with a $\mathrm{CO}_{2}$ absorbent, and the change in air volume corresponding to oxygen utilization by the animal recoded on a rotating spirometer drum specifically designed for small animals. The $\mathrm{VO}_{2}$ was expressed to $\mathrm{kg} \mathrm{BW}-0.75$ in the fed or fasted state as described by Klieber ${ }^{39}$ \& Wang et al., ${ }^{40}$ to adjust for differences in body weight and mass between lean and obese animals, as performed in our laboratory for many years. Measures of $\mathrm{VO}_{2}$ during cold exposure were obtained by moving the plexiglass canister from the $29^{\circ} \mathrm{C}$ to an ice water bath where the temperature was recoded to be $4^{\circ} \mathrm{C}$ throughout the 45 -minute period of cold exposure and without interrupting the closed-circuit circulation system during the process.

The thermic response to norepinephrine was determined by removing the animals from the thermogenesis cannister briefly to administer the NE in a single s.c. injection and immediately returning the animal to the chamber and allowing a few (1-3) minutes to reequilibrate. Animals were administered a fixed dose of $100 \mathrm{ug}$. of NE subcutaneously in the upper central dorsal region of the animal, and the peak thermic response obtained which typically occurred between 15 to 20 minutes post injection recorded. This dosage of NE has been observed to result in an optimal response and to be well tolerated by this and other strains of rat.

Statistical analysis was computed with the Minitab Statistics program (Minitab, Inc, State College PA). Descriptive statistics identified outliers within each group and values more than 2 standard deviations from the mean were eliminated from the statistical analysis. Overall variance was assessed by a 1 x 3 ANOVA and the additional interaction of diet on these responses by a $2 \times 2 \times 3$ ANOVA using the General Linear Model, a multiple regression program. Statistical significance was set at alpha of $\mathrm{p}<0.05$ with a Bonferroni correction where $\mathrm{p}=<0.02$ as described previously. ${ }^{14}$

\section{Results}

The body weights (BW) of lean and obese rats are depicted in Figure 1 and show that body weights of lean animals increased only gradually during the study ( $\mathrm{p}=\mathrm{N}$.S.), but the body weights of the obese phenotype were significantly greater at 4 months of age and continued to increase throughout the study, with an obese age effect of $p=<0.01$ and a lean vs. obese phenotype effect of $\mathrm{p}=0.00184$ (exact). The body weights of obese animals were $\sim 2$-fold greater than in lean animals at 4 months of age, $\sim 3$-fold greater at 14 months of age, and $\sim 3.5$ to 4 -fold greater at 24 months of age. Despite the excessive weight gain in the obese phenotype the animals remained non-diabetic throughout the ages studied in that there were no specific clinical signs noted that were consistent with the onset of NIDDM. ${ }^{14}$

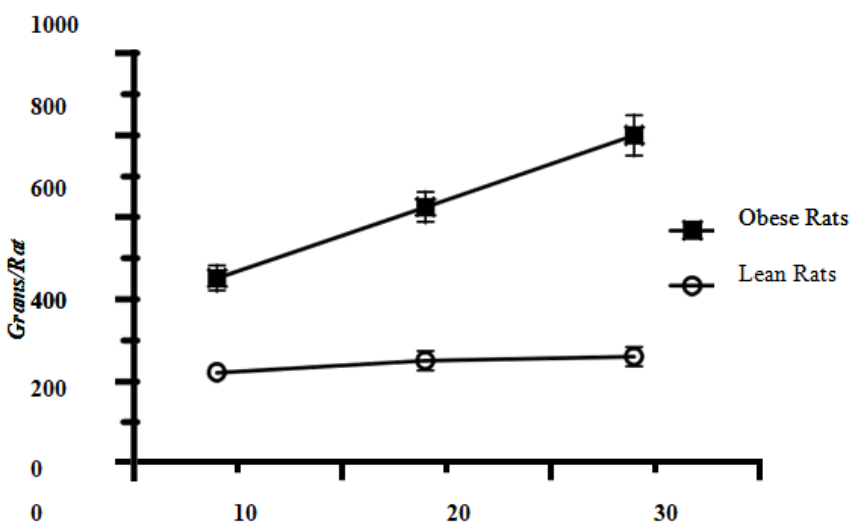

Months of age

Figure I Data are mean $+/$ - S.D., $N=12-20$ rats/group, $p=0.0018$ for lean vs obese phenotype.

The effects of lean and obese phenotype on resting VO2 are depicted in Figure 2 and show that the VO2 depicting RMR in the lean phenotype trended to decrease only modestly between 4 and 24 months of age. The RMR of lean phenotype was significantly greater than the RMR in the obese phenotype at all ages studied $(p=<0.05)$, and the downward trend of RMR in the obese phenotype became significant at 24 months of age $(\mathrm{p}=<0.05)$. The thermic effects of spontaneous cold exposure from $29^{\circ} \mathrm{C}$ to $4^{\circ} \mathrm{C}$ are depicted in Figures $3 \mathrm{~A} \& \mathrm{~B}$. In lean rats (Figure 3 (A), cold exposure resulted in a dramatic $\sim 5$-fold increase in the $\mathrm{VO} 2$ after 5 minutes cold exposure in 4-month-old lean rats. This dramatic increase in VO2 subsided to $\sim 2 \mathrm{x}$ RMR within 15 minutes and remained constant with increasing age with only a slight and non-significant downward trend thereafter in lean phenotype. In 14 months-old lean rats the increase in VO2 at 5 minutes averaged only a $\sim 4$-fold increase over RMR and returned to $\sim 2 \mathrm{x}$ RMR from 15 to 45 minutes cold exposure. In 24-month-old lean rats however, the cold induced increases in RMR were markedly diminished to only $\sim 3 x$ RMR at 5 minutes cold exposure but remained similar to the other lean age groups with a similar downward trend thereafter. In the obese phenotype, the peak responses at 5 minutes were $\sim 35 \%$ lower than observed in lean littermate rats at 5 minutes in 4- and 14-month-old rats with an age-associated downward trend. The thermic response to cold in the 24-month-old obese rats were recorded and were significantly impaired to only $\sim 40 \%$ of their lean littermates at the 5-minute interval and became further decreased with advancing age at all times measured. The thermic response of the oldest obese rats exposed to $4^{\circ} \mathrm{C}$ cold was significantly lower than in younger obese and in lean rats at all ages measured. Despite the cold stress applied, all animals of both phenotypes recovered from the environmental challenge uneventfully.

Administration of Norepinephrine (Figure 4A and 4B) at a standard dose of 100 ug NE subcutaneously resulted in a $\sim 1.5$-to1.6-fold increase in $\mathrm{VO}_{2}$ in the lean phenotype at all ages studied (Figure 4 (A)), while in obese rats depicted in Figure 4 (B) the NE administration resulted in a $\sim 1.4$-fold increase in $\mathrm{VO}_{2}$ at 4 months of age and with a statistically non-significant 1.1 to 1.2 -fold increases at the two older ages. In both phenotypes, the RMR and the NEstimulated $\mathrm{VO}_{2}$ responses both tended to decrease with increasing age and the NE responses remined lower and of lesser magnitude in obese than in lean rats throughout the study $(\mathrm{p}=<0.05$ for phenotype and age). 


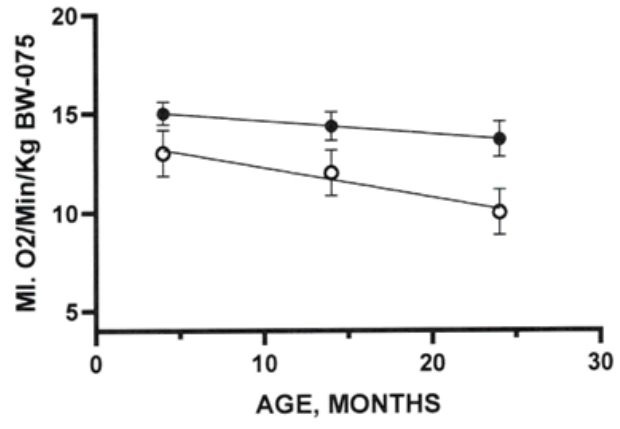

Figure 2 Effects of age and phenotype on RMR of fasting rats. data are mean+/- I SEM, $\mathrm{n}=6-12$ rats/group. $\mathrm{P}=<0.05$ for phenotype at 14 weeks, $\mathrm{p}-<0.0 \mathrm{I}$ at 24 months of age. ${ }^{14}$

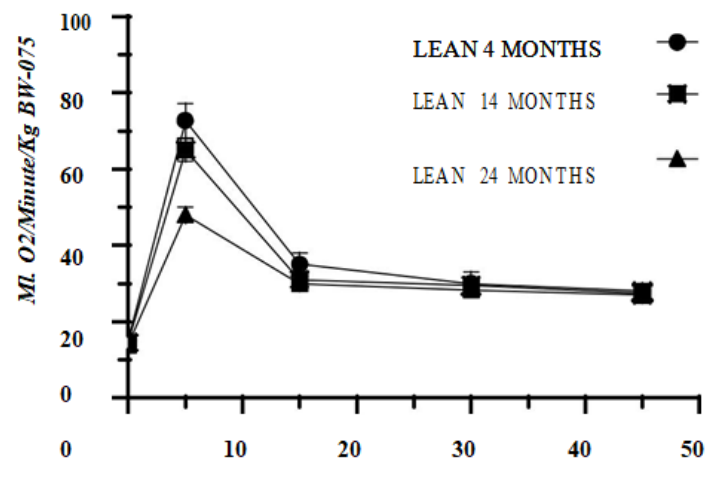

Minutes $4^{\circ} \mathrm{C}$ Cold Exposure

Figure 3 (A) Effect of cold exposure on $\mathrm{vo}_{2}$ in lean rats.

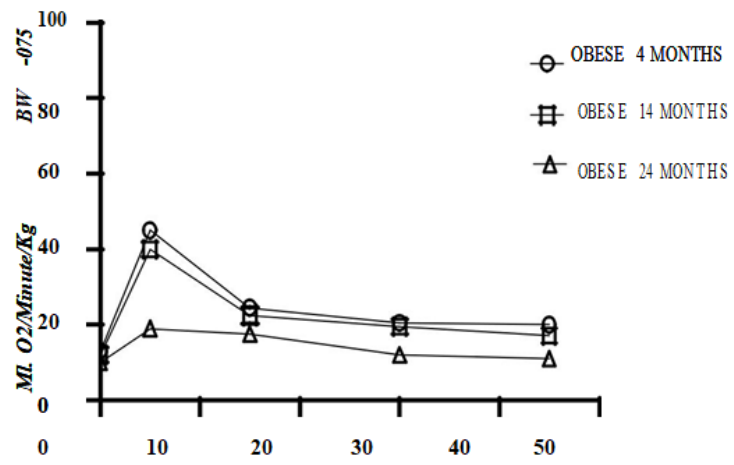

Minutes $4^{\circ} \mathrm{C}$ cold exposure

Figure 3 (B) Effects of cold exposure on $\mathrm{VO}_{2}$ in obese rats.

Figure $3 \mathrm{Effect}$ of $4^{\circ} \mathrm{C}$ cold on $\mathrm{VO}_{2}$ on lean rats (Figure $3 \mathrm{~A}$ ) and obese rats (Figure 3B), $n=6-12$ Rats/group. $P=<0.05$ for 24 vs 4 or 14 months of age at 5 minutes exposure. ${ }^{15}$

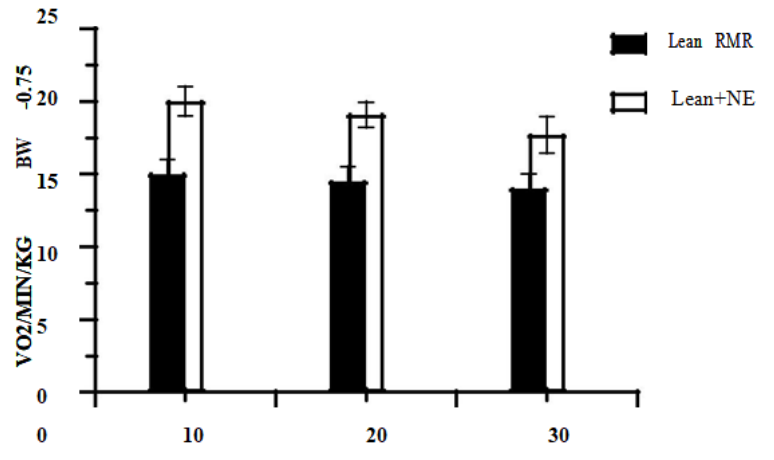

Months of age

Figure 4 (A) Effect of $\mathrm{NE}$ on $\mathrm{VO}_{2}$ of lean rats.

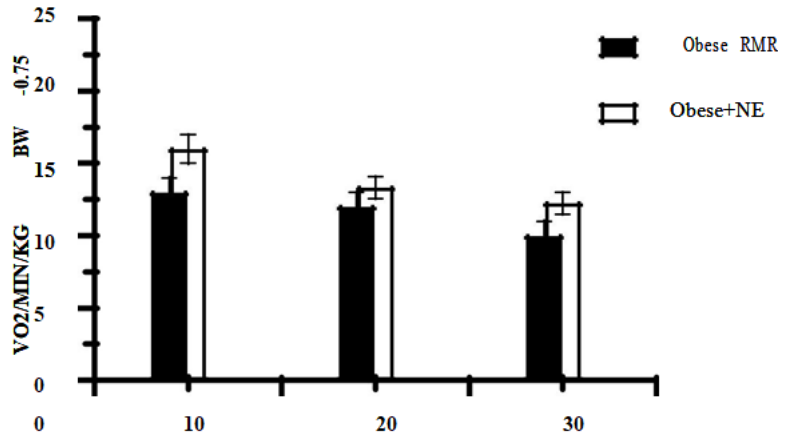

Months of age

Figure 4 (B) Effect of noradrenaline (NE) on $\mathrm{VO}_{2}$ in Lean (Figure 4 (A)) and obese (Figure $4(\mathrm{~B})$ ) rats, $\mathrm{n}=6-12$ rats/group. NE administered $100 \mu \mathrm{g} . /$ rat subcutaneous in upper central dorsal area and peak $\mathrm{VO}_{2}$ recorded I5-20 minutes post injection.

\section{Discussion}

The results of this study further characterize both the obesity and the thermic responses to cold environment and noradrenaline administration in the lean and obese phenotypes across much of the projected lifespan of this strain of rat. The results clearly show that animals bearing the obese phenotype become significantly heavier than their lean littermates at 4 months of age, and continue to increase in body weight thereafter, suggestive of a 'thrifty gene' mechanism associated with expression of the $-c p$ trait. ${ }^{21,22}$ In contrast, the body weights of animals bearing the lean phenotype remain relatively constant from 4 to 24 months of age, with only a minor and non-significant upward trend with advancing age, and while consuming the same standard stock rodent diet ad libitum as the obese phenotype throughout the study. No biochemical laboratory indices were examined during this study, ${ }^{41-47}$ but in other studies in this animal model and other rodent models bearing the same $-c p$ trait for obesity varying degrees of carbohydrate intolerance have been reported, with an apparent obesity linked development of NIDDM in the JCR:LA/N-cp, SKY/N $c p, \mathrm{WKY} / \mathrm{N}-c p$, and SHR/N-cp corpulent strains all of which share the same Koletsky ( $-c p)$ trait for obesity but expressed in different genetic backgrounds and with different degrees of backcrossing from the 5th to 12th or later backcrosses. From the studies reviewed, insulin resistance develops but full expression of the NIDDM trait appears to be conserved in the obese phenotype of this strain. ${ }^{8,13,14,36,37,48-51}$

A common observation among genetically obese rodent strains are impaired metabolic and thermic responses to diet and environment, likely due at least in part to metabolic defects in in the activation activity of BAT and possibly other thermogenic tissues, in combination with abnormalities in other endocrinologic and neuroendocrine parameters. Bukoweiki and Marette and others ${ }^{31-33}$ have reported an insulin dependent impairment in BAT thermogenesis in the obese phenotype of the SHR/Ntul//cp rat which shares the same genetic trait for obesity as the animals in the current study. While it is unclear from those studies if the impaired thermic responses are caused by a primary genetic factor, or a consequence of a secondary factor such as insulin resistance. The bulk of biochemical evidence suggests that the thermic defect may be caused by a secondary factor such as insulin resistance, rather than a primary genetically introduced mechanism in that all of the obese rodents' studies to date demonstrate impaired thermic responses to diet and environment, but not all share the same $-c p$ epigenetic trait for obesity. This suggests that other endocrinologic or neuroendocrine contributors may be implicated in the impaired thermogenesis reported. BAT when active is an active thermogenic 
tissue in mammalian species, and Himms-Hagen ${ }^{29}$ and others ${ }^{26,31-33}$ were among the first to have proposed that when active BAT tissue could function in a buffering and potentially energy wasting capacity in processes of energy balance during caloric excess. However, when BAT thermogenic activity becomes impaired due to endocrinologic or other factors, the defective BAT could contribute to permissive energy conservation and to the progressive development of obesity, regardless of the age of onset of the impaired thermogenic activity. In genetic animal models including the corpulent rat, the biochemical contributors to caloric efficiency begin soon after weaning at 21 days of age and become visually and physically noticeable before animals reach 6 weeks of age. ${ }^{8,14,17}$

Acute cold exposure might be expected to result in sharp and immediate increases in $\mathrm{VO}_{2}$, due initially at least in part to shiveringmediated heat generation, affecting primarily the muscle tissues where most shivering induced heat generation occurs. The initial cold induced response in $\mathrm{VO}_{2}$ is normally followed by rapid transition to heat production in BAT and perhaps other visceral organs including glucose uptake in liver \& Skeletal muscle, where cold stimulated catecholamine release would likely increase glycogen mobilization and subsequent glucose oxidation, further contributing to the heat generation. ${ }^{20}$ While shivering activity during cold exposure was not observed or quantified in the current study, it may have also contributed to the sharp increases leading up to the 5-minute mark of cold exposure, but it is proposed that transition to BAT thermogenesis was complete within 15 minutes and remained active thereafter. Any potential thermal protection afforded by the subcutaneous fat in the obese is likely minimal, as fat has been reported to be an inefficient insulator in thermal heat conservation due at least in part to the lesser blood flow in adipose vs muscular and visceral tissues. ${ }^{8,23}$

Impaired thyroidal activity at the local (cellular or intracellular level) is also a likely contributor to the development of an obese state in this animal strain. Numerous reports indicate that the normal thyroidal responses to diet and environment are impaired in the obese phenotype of this and other obese rodent strains. Tulp and Shields reported that thyroidal and thermic responses to cafeteria overfeeding were diminished in the obese phenotype of the LA/Ntul//-cp rats ${ }^{49}$ and McKee and Tulp reported that T3 neogenesis from T4 was likewise decreased in the obese phenotype of this strain ${ }^{41}$ and Paz Nava et al and others reported that the T4 half-life was prolonged in the obese phenotype of both LA/Ntul//-cp and SHR/Ntul//-cp rats, indicative of decreased T4 5' deiodinase activity in peripheral tissues. ${ }^{21,27,43}$ Obese Zucker rats, which share a different genetic origin of their obesity have also been shown to exhibit an impaired thyroidal and thermogenic response to diet compared to their lean littermates. ${ }^{31,44}$ Decreases in thyroidal activity have long been implicated in conservation of energy expenditure where decreases in T3 activity at the organ and tissue level may contribute to aberrations in elements of $\mathrm{CHO}$ and protein metabolism, consistent with a progressive development of improved caloric efficiency and excess energy storage and resulting adiposity. York et al., ${ }^{45}$ has reported similar aberrations in thyroid hormone metabolism and receptor biding in obese (ob/ob) mice which also express a recessive genetic trait for obesity analogous to the expression of the $-c p$ and fatty $(-f a)$ traits in rats. ${ }^{8,43-45}$

Triiodothyronine (T3) availability, when elevated can trigger increases in the rate of protein turnover via increases in protein degradation and ensuing corresponding changes in protein synthesis, commonly referred to as protein turnover and closely linked to overall $\mathrm{N}$-balance of an organism. ${ }^{23}$ Protein synthesis, at 4 high-energy phosphate bonds consumed per peptide bond formed represents one of the most energetically expensive biochemical processes of cells and tissues and has also been shown to be decreased in the obese phenotype of the $-c p$ rat $^{24}$ Other factors including the hepatic T3 dependent a-glycerophosphate shuttle of mitochondria for generation of reducing equivalents needed for optimal mitochondrial thermic responses become decreased proportional to circulating T3 concentrations ${ }^{16,46}$ resulting in a shift from an energy wasting metabolic cycle to the extranuclear, extramitochondrial and energy conserving MalateAspartate shuttle needed for lipid biosynthesis and fatty accretion in adipose tissue. Thus, when circulating concentrations of T3 become elevated, RMR and the thermic responses to diet and environment may become increased and thermogenesis may also become increased in proportion to the changes in T3 availability. In contrast, when T3 availability becomes limiting, biochemical energy expenditure may become decreased, and become contributory epigenetic mediated factors in the development of obesity. ${ }^{22}$

The biochemical parameters of the impaired thermic responses to cold exposure and noradrenaline administration in the obese phenotype were not reported in the current study, but laboratory findings from other studies in this strain suggest that both insulinogenic and thyroidal factors were likely contributory. The results clearly demonstrate that the expression of the $-c p$ trait results in a marked development of extreme obesity in the obese phenotype, and which is progressive throughout much of the expected life span of this strain. Moreover, when lean littermates were fed the same diet for the same duration under the same environmental conditions, often sharing the same cages, overall energy balance was maintained, with only a modest and non-significant trend toward any increases in body weight or adiposity with advancing age. Measures of resting, noradrenaline- and cold induced thermogenesis occurred normally in the lean phenotype throughout the reported duration of study, where robust thermic responses were reproducibly observed. The effects of aging to 24 months of age on the thermic parameters in lean animals were minimal at best and failed to be statistically significant. In contrast, the obese phenotype, expressing the $-c p$ trait demonstrated measurable decreases in the capacity for cold and NE-stimulated thermogenesis, and which parameters became further compromised with advancing age, and corresponding with the decreases in RMR observed in aging obese rats. The development of obesity in animals that expressed the obese phenotype is likely a direct consequence of the expression of the genetic $-c p$ trait for obesity, since in this strain non- $c p$ linked variances between lean and obese animals were minimal if at all due to the congenic nature of the animal model. In contrast the impaired thermic responses to obesity during aging in this strain are likely the consequence at least in part to the impaired insulinogenic and thyroidal influences reflected in their metabolic parameters secondarily linked to the expression of the $-c p$ trait. The development of insulin resistance is well established in obesity in both man and animals, and observations of caloric efficiency in the obese phenotype reported elsewhere are compatible with insulin resistance being a key contributing factor in the impaired thermogenesis in the present study. Multiple hormonally mediated metabolic processes are likely contributory, with the metabolic sequelae of insulin resistance being a primary suspect factor not only in the caloric efficiency of weight gain leading to obesity but also in the expression of the thermogenic parameters leading to the development and maintenance of obesity throughout much of the projected lifespan of the corpulent rat.

\section{Acknowledgments}

The author wishes to acknowledge the contributions of Dr. Susan DeBolt, Mr. Huang Peisong, and Dr. Stephen Dubin, of Drexel University and the late Dr. Otho E Michaelis IV, of the 
Carbohydrate Nutrition Research Laboratory, Beltsville, MD, USDA for contributions, data collection and management assistance in completion of this research and UHHVI Professor Dr. Aftab R Awan for prepublication editorial review.

\section{Conflicts of interest}

No conflict of interest was reported by the authors.

\section{Funding}

None.

\section{References}

1. Jacks DG, Kerna NA. A Comprehensive Analysis of Obesity Part 1 . Overview of Obesity. J Obese Nutr Disord. 2018.

2. Haffner SM. Sex hormones, obesity, fat distribution, type 2 diabetes, and insulin resistance: epidemiological and clinical correlation. Int $J$ Obese. 2000;24:S56-S58.

3. Rotondi M, Magri F, Chiovato L. Thyroid and Obesity: Not a OneWay Interaction. The Journal of Clinical Endocrinology \& Metabolism. 2011;96(2):344-346.

4. Rahman M. PhD Thesis: A Systems-Based Review of Adipose Tissue as an Organ: A model of autonomic, immunological, and endocrine influences. USAT Montserrat, BWI; 2018. 258 p.

5. Ravussin EM, Bogardus C, Schwartz RS, et al. Glucose induced thermogenesis and insulin resistance in man. Int J Obesity. 1985;9:103109.

6. Jacks DG, Kerna NA. A Comprehensive Analysis of Obesity Part 1. Overview of Obesity. J Obese Nutr Disord. 2018.

7. Haffner SM. Sex hormones, obesity, fat distribution, type 2 diabetes, and insulin resistance: epidemiological and clinical correlation. Int $J$ Obese 2000;24:S56-S58.

8. Resources Branch. National Institutes of Health, Bethesda, MD. 1988. p. 7-10.

9. Michaelis OE. IV in: New Models of Genetically Obese Rats for Studies in Diabetes, Heart Disease, and Complications of Obesity, Veterinary Resources Branch, Division of Research Services, NIH publication, Bethesda; 1988. p. 13-15.

10. Greenhouse DD, Hansen CT, Michaelis OE IV. Development of Fatty and Corpulent Rat Strains. In: New Models of Genetically Obese Rats for Studies in Diabetes, Heart Disease, and Complications of Obesity. NIH publication, Division of Research Services, Veterinary Resources Branch, National Institutes of Health, Bethesda; 1988. 36 p.

11. Tulp OL, Brown T. Effect of a Fructan-Chromium Complex on Glycemic Responses of Congenic Obese LA/Ntul/ /-cp Rats. J Nutr Health Food Eng. 2016;5(2): 594-598.

12. Huang HJ, Young AA, Koda JE, et al. Hyperamylinemia, hyperinsulinemia, and insulin resistance in genetically obese LA/N-cp rats. Hypertension. 1992;19(1 Suppl):101-109

13. Tulp OL. Sympathetic and Thyroidal Components of Diet-Induced Facultative Thermogenesis in the LA-Corpulent Rat. Brain Res Bull. $1985 ; 348$.

14. Michaelis OE, Ellwood KC, Tulp OL, et al. Effect of feeding sucrose or starch diets on parameters of glucose tolerance in the LA/N-corpulent rat. Nutr Res. 1986;6(2):95-99.

15. Tulp OL. Effects of Aging, Phenotype and Carbohydrate Feeding on Caloric Efficiency and Adiposity in the LA/Ntul/l-cp (Corpulent) Rat. $A d v$ Obes Weight Manag Control. 2021:11(1)5-11.
16. Tulp OL. Impaired peripheral thyroid activity, decreased protein turnover, and development of obesity in the LA-corpulent rat. Prog X111 Int Cong Of Nutr. 1985.

17. Galton DA, Bray G. Metabolism of a-Glycerol Phosphate in Human Adipose Tissue in Obesity. The Journal of Clinical Endocrinology \& Metabolism. 1967;27(11):11.

18. Tulp OL, DeBolt SP. Metabolic and thermic responses to diet and cold environment $\left(4^{\circ} \mathrm{C}\right)$ in obesity during aging. In: Mini Nutritional Assessment (MNA) in the Elderly: Research and Practice. $44 \mathrm{p}$.

19. Tulp, OL, Awan AR, Einstein, GP, et al. Enhanced caloric efficiency contributes to adiposity in the LA/Ntul//-cp rat. Faseb J. 2021;35:S1.

20. Danforth E Jr. The role of thyroid hormones and insulin in the regulation of energy metabolism. Am J Clin Nutr. 1983;38:1006-1017.

21. Tulp OL. Impaired activation of thermogenesis in the corpulent rat. Life Sciences. 1984;35:1699-1705.

22. Granner DK. The Diversity of the Endocrine System, Harpers Illustrated Biochemistry. In: Murray RK, editor. 2003;42:437-439.

23. Tulp OL, Paz Nava M, Black DE, et al. Genetic determinants of T3 receptor affinity and thermogenesis in LA/N-cp rats" Proceedings, XV International Congress of Nutrition Adelaide. International Union of Nutritional Sciences. 1993. 481 p.

24. Tulp OL. Expression of peripheral thyroxine 5'deiodinase activity: A genetic link between the metabolic efficiency of under nutrition and malnutrition and the predisposition toward the development of obesity. Prog. 11 Inter American conferences on health education. J International Union of Health Sciences. 1984

25. Granner DK. Protein Synthesis \& the Genetic Code. Harpers Illustrated Biochemistry. In: Murray RK, editor. 2003;38:364-371.

26. Tulp OL. Decreased protein synthesis and degradation in early obese LA corpulent rats. Fed Proc. 198544(4):1501.

27. Zhao JB, Cannon B, Nedergaard J. Thermogenesis is beta3- but not betaladrenergically.mediated in rat brown fat cells, even after cold acclimation. Am J Physiol. 1998;275(6):R2002-11.

28. Tulp OL, Black DE, Outrim D, et al. Peripheral thyroid hormone metabolism and actions (THMA) in lean and obese LA/N -cp rats. FASEB J. 1994

29. Lisboa PC, Passos MCF, Dutra SCP, et al. Increased 5' iodothyronine deiodinase activity is a maternal adaptive response to protein restriction during lactation. Journal of Endocrinology. 2003;377:261-267.

30. Himms-Hagen J. Thermogenesis in BAT as an energy buffer. NEJM. 1983;311:1150-156.

31. Tulp OL, Q Chen, P Tso. Nonshivering thermogenesis and metabolic parameters of obese rats after fetal hypothalamus implants. Proceedings. 16th Int Congress on Nutrition, Montreal Que. 1997. 160 p.

32. Young RA, Tulp OL, Horton ES. Thyroid and growth responses of young Zucker obese and lean rats to a low protein-high carbohydrate diet. J Nutr. 1980;110:1421-1431.

33. Stock MJ, Rothwell N. Diet Induced thermogenesis and energy flux through brown adipose tissue. In: Proceedings of the XII International Congress of Nutrition. In: Taylor TG, Jenkins NK, John Libbey, editors. London; 1985. p. 321-325.

34. Marette A, Tulp OL, Bukowiecki LJ. Mechanism linking insulin resistance to defective thermogenesis in brown adipose tissue of obese diabetic SHRN -cp rats. Int J Obese. 1991;15:23.

35. Marette A, Maurige P, Bukowiecki LJ, et al. Hormonal regulation of brown adipocyte glucose transport in genetic obesity. In: Obesity: Dietary factors and Control. In: DR. Romsos, editor. Basel: Karger. 1991. p. 119130. 
36. Abdoulaye D, Pierce WD, Kelly SE, et al. Abnormal insulin and glucose metabolism in theJCR:LA-corpulentrat. Front Nutr. 2016;3:44.

37. Vedula U, Schnitzer-Polokoff R, Tulp OL. The effect of acarbose on the food intake, weight gain, and adiposity of LA/N-cp rats. Comp Biochem Physiol A Comp Physiol. 1991;100:477-482.

38. Tulp OL, Stevens C, Barbie OA. Non-shivering thermogenesis and obesity in the adult diabetic Wistar fatty rats. Comp Physiol Pharm. 1989;92A(1):37-41.

39. Tulp, OL, Michaelis OE IV, Servetnick, DA, Hansen, CT, and Black, DE. Nonshivering thermogenesis and obesity in the diabetic SKY/N - cp rat Comp Biochem Physiol. 1994;107A:195-201,

40. Michaelis OE, Ellwood KC, Tulp OL, et al. Effect of feeding sucrose or starch diets on parameters of glucose tolerance in the LA/N-corpulent rat. Nutr Res. 1986;6(2):95-99.

41. Kleiber M. The Fire of Life: An Introduction to Animal Energetics. Wiley publishers. New York NY USA; 1961.

42. Wang ZM, Zhang J, Ying Z, et al. Organ-Tissue Level Model of Resting Energy Expenditure Across Mammals: New Insights into Kleiber's Law. Int Scholarly Research Network, IBSN Zoology. 2012; ID 673050.

43. Tulp OL. Thermogenesis and Obesity in the LA/N-cp and SHRN -cp Rat Strains. in New Models of Genetically Obese Rats for Studies in Diabetes, Heart Disease, and Complications of Obesity, Veterinary Resources Branch, Division of Research Services, NIH Publication, Bethesda MD, 1988. p. 17-25.
44. Tulp OL, McKee, STD. Triiodothyronine (T3) neogenesis in lean and obese LA/N-cp rats. Bioch Biophys Res Commun. 1986;140:134-142.

45. Young RA, Shih-Lieh F, Prosky J, et al. Hepatic Conversion of thyroxine in obese and lean Zucker rats. Am J Physiol. 1987;252:E63-E67.

46. York DA, Otto W, Taylor TG. Thyroid status of obese (ob/ob) mice and its relationship to adipose tissue metabolism. Comparative Biochemistry and Physiology Part B: Comparative Biochemistry. 1978;59(1):59-65.

47. Tatleman HM, Tyzbir RS, Tulp OL. Effects of overfeeding on BAT and liver mitochondrial metabolism and shuttle activity in adult rats. Fed Proc. 1981;40:871.

48. Tulp OL. Expression of peripheral thyroxine 5'deiodinase activity: A genetic link between the metabolic efficiency of undernutrition and malnutrition and the predisposition toward the development of obesity. Prog. 11 Interamerican conference on health education. $J$ Int Union of Health Sciences. 1984.

49. Kahle EB, Dadgari JM, Dudley GA, et al. Adaptive Response of Enzymes of Carbohydrate and Lipid Metabolism to Exercise, in New Models of Genetically Obese Rats for Studies in Diabetes, Heart Disease, and Complications of Obesity. Veterinary Resources Branch, Division of Research Services, NIH publication,Bethesda, MD. 1988. p. 143-148.

50. Tulp OL, SJ Shields. Thermogenesis in cafeteria-fed LA/N-cp (Corpulent) rats. Nutr Res. 1984;4:325-332.

51. Tulp OL, DeBolt SP, Einstein, GP. Decreased capacity for thermogenesis during afing in lean and obese LA/Ntul//cp (corpulent) rats. Faseb J. 2021;35(1). 